\title{
On-Chip Electrochemical Impedance Spectroscopy for Biosensor Arrays
}

\author{
Chao Yang, Daniel Rairigh, Andrew Mason \\ Department of Electrical \& Computer Engineering \\ Michigan State University, East Lansing, United State \\ \{yangchao, rairighd, mason\}@egr.msu.edu
}

\begin{abstract}
Electrochemical impedance spectroscopy (EIS) is a powerful tool for characterizing biological materials, including lipid bilayers and many membrane proteins. However, traditional EIS methods are very slow at low frequencies, where these materials respond in biosensor applications. To enable dense arrays of biosensors based on tethered bilayer lipid membranes (tBLM), a new approach for EIS has been developed. This paper introduces a methodology and circuit that can rapidly perform EIS in the $1 \mathrm{mHz}$ to $100 \mathrm{kHz}$ frequency range. A circuit implementing this new approach has been realized in $0.5 \mu \mathrm{m}$ CMOS technology with 3.3 voltage power supply. In the sub-hertz range where membrane protein biosensor response is most critical, the circuit can measure impedance with 8 bit resolution in $20 \mathrm{~ms}$, three orders of magnitude faster than traditional integrator-based circuits. Though tailored for the low frequency spectrum in biosensor applications, the EIS circuit can be used to measure impedance in a wide range of sensory materials.
\end{abstract}

\section{INTRODUCTION}

Protein modified tethered bilayers lipid membranes (tBLM) [1] provide a means to measure, with high specificity and high sensitivity, unique biochemical analytes and biological phenomena. They hold great promise in many applications of biotechnology including diseases diagnosis and environmental monitoring. Electrochemical impedance spectroscopy (EIS) techniques are the best means to interrogate many tBLM-based biosensors. Currently, benchtop instruments are used to perform EIS [2,3], and no chipscale EIS systems exist.

These biosensors offer their greatest potential when used in high-density arrays of tBLMs modified with different proteins, which encourages the development of compact systems. Two constraints impede the further progress in the miniaturization of tBLM-based biosensor arrays [4]. First, routing hundreds of raw signals off the array chip introduces bandwidth challenges as well as performance-limiting noise; thus on-chip instrumentation capable of processing and reducing the raw data is necessary to fully enable these arrays. Second, the measurement speed of traditional EIS methods is not adequate to interrogate a high density array rapidly enough to ensure all important data is recorded.

This paper described an on-chip EIS system targeting these constraints. In section II, the characteristics of the

This work was supported in part by the Engineering Research Centers Program of the National Science Foundation under Award Number EEC9986866.
tBLM biosensor and its interrogation method are discussed. In section III, a new measurement scheme targeting highdensity tBLM based biosensor arrays is introduced, and its circuit realization is presented in section IV.

\section{TBLM BASED BIOSENSORS}

\section{A. Protein modified tBLM and equavalent circut model}

A tBLM is a synthetic lipid membrane that is tethered to the surface of a solid electrode. Proteins, including ion channels as shown in Fig. 1(a), can be embedded in the tBLM to mimic the function of a cell membrane or immobilize the protein for characterization. Only very specific analytes will cause these channels to open and allow ions to pass through them. The open/closed state of the ion channels reflects the presence of a certain analyte in the solution, and the impedance across the membrane corresponds to the analyte concentration. Thus, the proteinmodified tBLM can serve as a very sensitive and selective sensor element. tBLM based biosensors modified by different proteins can be formed into an array to detect and measure a wide range of analytes efficiently.

To measure a tBLM biosensor electrically, an equivalent circuit model is used as shown in Fig. 1(b). The two ends of this equivalent circuit present the solution side and solid electrode side of the tBLM biosensor. $R_{S}$ represents the serial resistance of the solution. $C_{d l}$ represents the double layer capacitance of the electrode metal-solution interface. $C_{M}$ is the capacitance of the lipid bilayers. $R_{M}$ is the resistance of the lipid bilayer, which is normally much larger than the

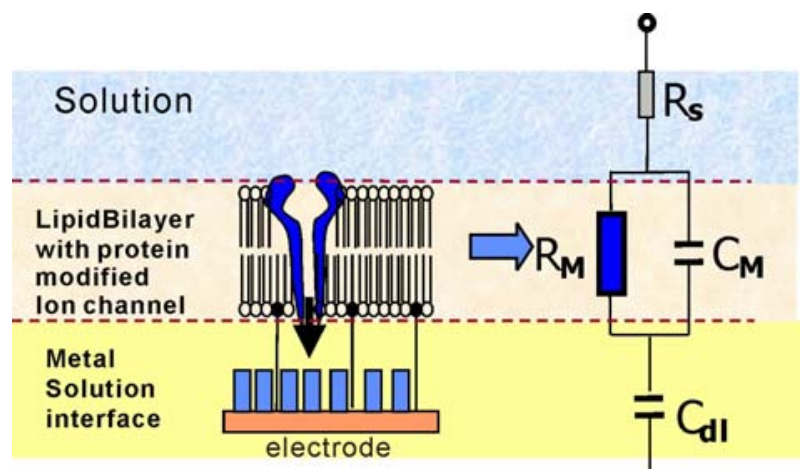

(a)

(b)

Figure 1. (a) Structure and (b) equivalent circuit model of a tBLM-based biosensor with an ion channel protein. 
solution resistance $R_{s}$. The states of the ion channel proteins change the lipid bilayer resistance. Therefore $R_{M}$ relates directly to the analyte concentration in the solution.

\section{B. Interrogation of $t B L M$ based biosensor}

In measuring analyte concentrations, the most important component in the equivalent circuit model is $R_{M}$. However, $R_{M}$ cannot be measured directly by applying a DC voltage or current, because $C_{d l}$ blocks the DC path. Instead, electrochemical impedance spectroscopy (EIS), which measures the impedance over a certain frequency range, is employed to acquire the overall impedance response of the tBLM biosensor. Because $R_{M}$ dominates the response within a certain frequency range (roughly $0.1-10 \mathrm{~Hz}$ for tBLM sensors), the value of $R_{M}$ can be extracted from the impedance response. Typically, admittance (1/impedance) is measured rather than impedance because it is generally easier to apply a voltage stimulus and read the current than the reverse. If needed, impedance values can always be calculated from admittance data.

There are two common methods for making EIS measurements. Many systems stimulate the sensor with a pulse and then process the response using the Fast Fourier Transform (FFT) algorithm [5]. This is not practical for highdensity arrays as the digital signal processing (DSP) blocks necessary to implement the FFT consume a lot of space, and as noted earlier, routing hundreds of raw signals off-chip for external processing is also not ideal. The other common method is called a Frequency Response Analyzer (FRA). This system stimulates the sensor one frequency at a time and then processes the response using multiplication and integration. The drawback of this approach is that an integrator needs operate over several periods of the excitation signal to filter out the AC interference. As a result, FRA is extremely slow $\left(\sim 10^{4} \mathrm{sec}\right)$ when measuring the low frequency range, thus limiting its application for tBLMbased biosensor arrays.

\section{NEW INTERROGATION METHOD}

To enable on-chip membrane protein biosensor arrays, both the size and the speed of the measurement system is critical. The traditional FRA method can be compact if realized on chip, but suffers from a long settling time for low frequency interrogation. To overcome this problem, a new EIS method had been developed to dramatically improve measurement speed and provide a response time that is independent of measurement frequency.

\section{A. Mathematical model of new method.}

In contrast to tradition impedance measurement methods, the new method quickly extracts complex (real and imaginary) data by simultaneously characterizing two identical sensor elements. AC interference is canceled automatically by analog processing of the complex signal. The system level diagram is shown in Fig. 2. Two identical sensor elements are interrogated at the same time using sinusoid stimuli with a $90^{\circ}$ phase shift. By processing the stimuli and response in the complex domain (dealing with

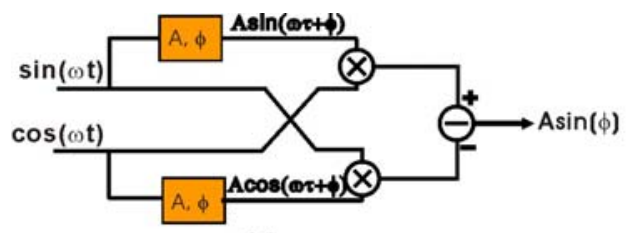

(a)

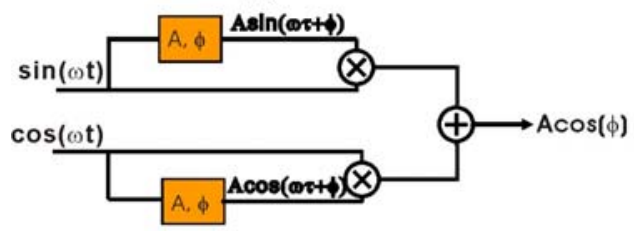

(b)

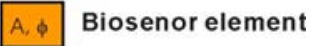

Figure 2. Signal flow diagram of new EIS method that generates (a) the imaginary portion of admittance and (b) the real portion of admittance.

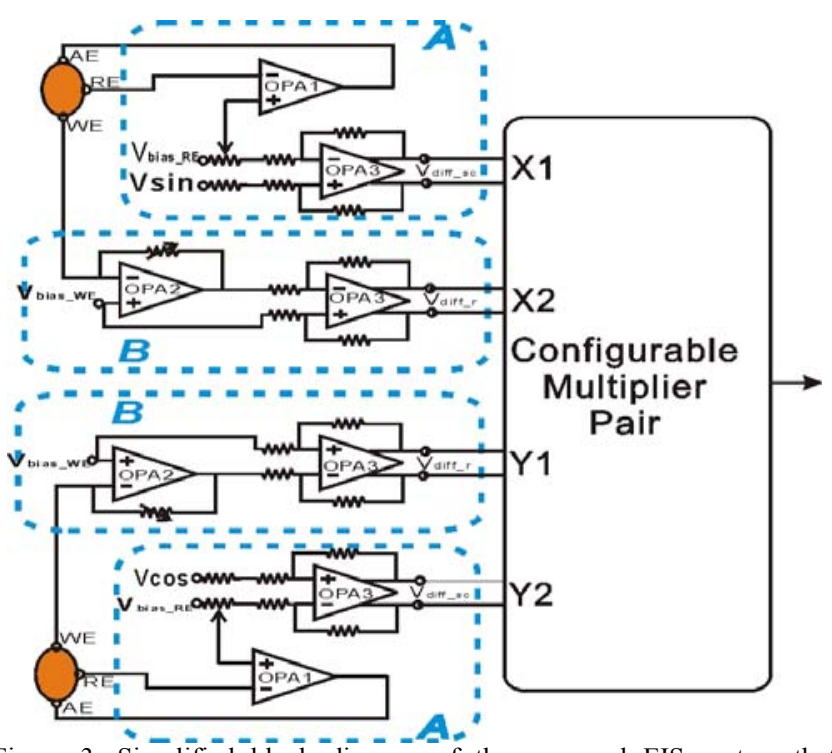

Figure 3. Simplified block diagram of the proposed EIS system that realizes the method shown in Fig. 2. Blocks labeled A convert stimulus to a differential signal and add DC offset. Blocks labeled B convert sensor response to a differential signal.

the real and imaginary signals), the real portion and imaginary portion of the admittance are computed directly at the output without $\mathrm{AC}$ interference. Assuming that a biosensor has a current response to a sinusoidal voltage stimulus at frequency $\omega$ given by

$$
A \sin (\omega t+\varphi)=f(\sin (\omega t))
$$

where $A$ is the amplitude and $\varphi$ phase response, then the real and imaginary portions of its admittance are $\operatorname{Acos}(\varphi)$ and $\operatorname{Asin}(\varphi)$, respectively, which can be derived from:

$$
\begin{aligned}
& A \sin (\varphi)=A \sin (\omega t+\phi) \cdot \cos (\omega t)-A \cos (\omega t+\phi) \cdot \sin (\omega t) \\
& A \cos (\varphi)=A \sin (\omega t+\phi) \cdot \sin (\omega t)+A \cos (\omega t+\phi) \cdot \cos (\omega t)
\end{aligned}
$$

The signal flows in Fig.2 implement (2) and (3). The advantage of this representation is that the output is only a $\mathrm{DC}$ value; all AC components are canceled automatically. 


\section{B. Implementation of the new method.}

To apply this new method to the three-electrode measurement system widely used in electrochemical interrogation, the system in Fig. 3 was developed. Two identical biosensors are interrogated simultaneously in this system. A configurable multiplier pair performs the signal processing shown in (2) and (3) in the analog domain and produce the real or imaginary portion of admittance depending on the configuration. The circuits in area $A$ have two functions: 1) converting the stimulus signal from single ended form to differential signal for differential inputs of the multiplier pair, and 2) applying proper DC bias and transferring the AC excitation for the sensor. The current response of the sensors is converted into differential voltage for the multiplier pair within the circuit in area $B$.

\section{Circuit REAlizATION}

\section{A. Configurable multiplier pair block}

The multiplier pair realizes the subtraction or summation of two products to perform the analog signal processing described by (2) and (3). A modified CMOS four-quadrant analog multiplier was designed as shown in Fig. 4. Its inputs are differential voltages and its output is a differential current. The four analog multipliers in this circuit generate the four different products in (2) and (3). Ctrl configures the circuit to perform (2) or (3). In either configuration, only two products are needed at any given time, leaving two of the multipliers in an idle state. Although these idle multipliers require more chip area, they avoid the linearity degradation associated with switching the input voltage signal path to reuse the multiplier. Switches at the output generate negligible nonlinearity because the output is a current. To achieve 8-bit performance for the whole system, the multiplier pair must provide more than 8-bits of resolution.

\section{B. Interfacing and signal conditioning circuit}

To implement (2) and (3), the multiplier pair needs both the input excitation signal and the current response signal of the sensor. However, the properties of the raw excitation and sensor response signals are not ideally suited for analog processing. The sensors respond well to small excitation signals and have a current-mode output, and all inputs and outputs are single ended. In contrast, the multiplier pair prefers large differential signal inputs in order to achieve good linearity and minimize the effect of noise and offset. The circuits shown in areas A and B in Fig. 3 realize the interfacing and signal conditioning necessary to bridge this difference.

For the circuits in area $A$ of Fig. 3, OPA3, a differential op-amp, is employed to convert the single-ended stimulus to differential form. To minimize the effects of the noise and offset of the OPA3, the amplitude of the input stimulus is set large enough for use by the multiplier. To generate a small amplitude stimulus for the biosensor, the signal is derived from the single-to-differential converting circuit through the tip of a variable resistor. It is fed to the positive input of OPA1, which forms a feedback loop with the sensor to apply the proper excitation based on the signal at its positive input. OPA1 drives the auxiliary electrode of the tBLM, which could be a large capacitive load in the nanofarad range [1]. The stability of the loop is very critical. Thus, OPA1 is designed with a cascoded folding structure with a source follower as the output stage. It can provide enough gain and good phase margin with large capacitive loading.

The circuits in area $B$ are designed to bridge the current output of the biosensor and the differential voltage input of the multiplier pair. Two stages are used. The first stage realizes the conversion from current into voltage. The conversion gain is tunable through a variable resistor in the feedback loop to adapt to the impedance of the sensor and provide a large output swing for the multiplier pair to achieve better accuracy. The second stage finishes the singleended-to-differential conversion.

OPA3 is used for single-to-differential conversion in both of the circuits in areas $A$ and $B$. However, the common mode voltages for these two cases are different, so OPA3 was designed to support a large common mode swing.

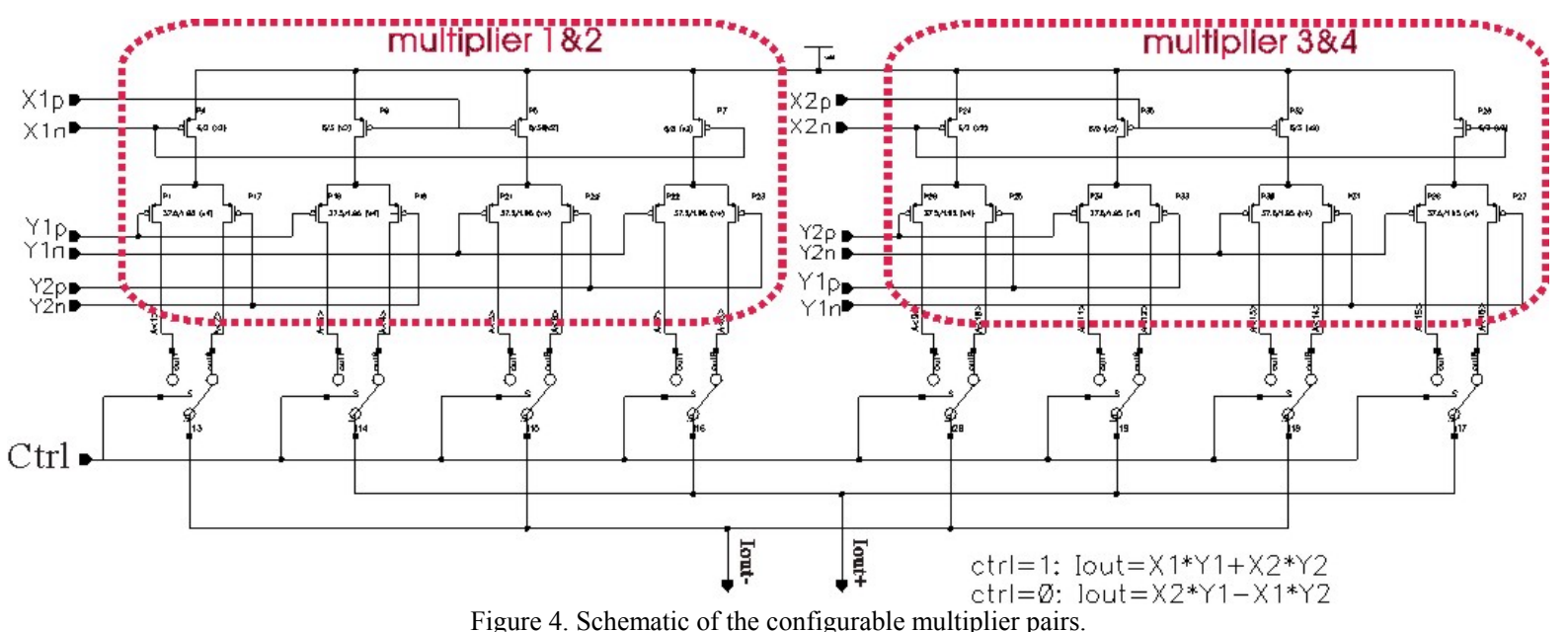




\section{Simulation Result}

The on-chip EIS system was implemented in $0.5 \mu \mathrm{m}$ CMOS technology and occupies $1.2 \mathrm{~mm}$ x $0.6 \mathrm{~mm}$ (Fig. 5). It consumes $1.8 \mathrm{~mA}$ with a $3.3 \mathrm{~V}$ supply. A dedicated area with metal contacts is reserved for post CMOS fabrication of the electrode array and subsequent formation of bio-interface layers. The electrode array can be expanded over the entire chip surface without impacting the design or performance of the EIS circuit.

Post layout simulations of the multiplier pairs show they can provide a linearity of at least $56 \mathrm{~dB}$ for any combination of input signals, with peak-to-peak swings ranging from $100 \mathrm{mVpp}$ to $800 \mathrm{mVpp}$ (maximum). $56 \mathrm{~dB}$ assures that an overall performance of 8 bits is not limited by linearity.

To evaluate the overall performance of the on-chip EIS system, simulations were run at different frequencies using the biosensor model in Fig. 1. Figure 6 shows the admittance of the Fig. 1 biosensor model, and Fig. 7 shows the simulated results from the new EIS circuit for the same model. Two values of $R_{M}$ are plotted to illustrate the sensors operational response. The similarity of Fig. 6 and Fig. 7 shows that the changes on the biosensor are well tracked by the EIS circuit. A statistical comparison summarized in Table 1 shows that the correlation coefficient between the two plots is high. Using linear regression to map the output to admittance, the average percentage error was about $2.65 \%$. The error is expected to reduce further with higher order regression and further circuit optimization.

While integrator based EIS systems have a response time proportional to the excitation signal period, this system is independent of the excitation frequency and the response time was measured to be less than $20 \mathrm{msec}$. In the sub-Hertz range, this provides three orders of magnitude in speed improvement, guaranteeing a very fast measurement, which is critical for readout of high-density arrays.

Table 1. Correlation of measurement to the model

\begin{tabular}{|c|c|c|c|c|c|}
\hline & \multicolumn{2}{|c|}{$R_{M}=50 \mathrm{~K}$} & \multicolumn{2}{c|}{$R_{M}=500 \mathrm{~K}$} & $\begin{array}{c}\text { Average } \\
\text { Performance }\end{array}$ \\
\hline & $\begin{array}{c}\text { Real } \\
\text { portion }\end{array}$ & $\begin{array}{c}\text { Imaginary } \\
\text { portion }\end{array}$ & $\begin{array}{c}\text { Real } \\
\text { portion }\end{array}$ & $\begin{array}{c}\text { Imaginary } \\
\text { portion }\end{array}$ & \\
\hline Correlation & 0.9998 & 0.99988 & 0.9998 & 0.9998 & 0.9998 \\
\hline $\begin{array}{c}\text { Average } \\
\% \text { error }\end{array}$ & $-2.68 \%$ & $-2.72 \%$ & $4.08 \%$ & $1.11 \%$ & $2.65 \%$ \\
\hline
\end{tabular}

\section{CONCLUSION}

A new on-chip electrochemical impedance spectroscopy system was designed for rapid interrogation of on-chip biosensor arrays. Its response time for the $1 \mathrm{mHz}$ to $100 \mathrm{kHz}$ frequency range is less than $20 \mathrm{msec}$. This design has been fabricated with AMI $0.5 \mu \mathrm{m}$ CMOS process. Simulation shows that it can provide 8 bits resolution while operating a thousand times faster than the traditional method for low frequency measurement.

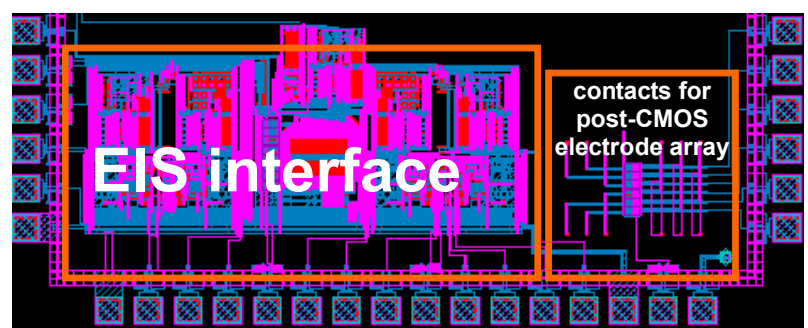

Figure 5: Layout snapshot of the on-chip EIS system (currently being fabricated).

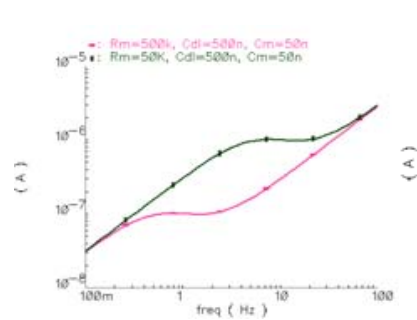

(a)

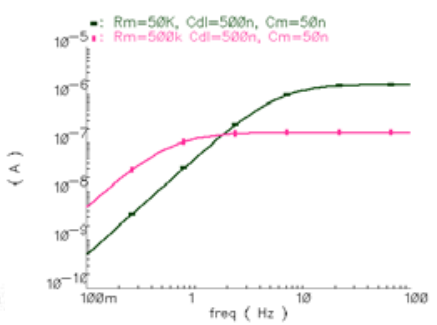

(b)
Figure 6. Admittance characteristics of a model tBLM biosensor with different values of $\mathrm{R}_{\mathrm{M}}$, (a) imaginary portion (b) real portion.

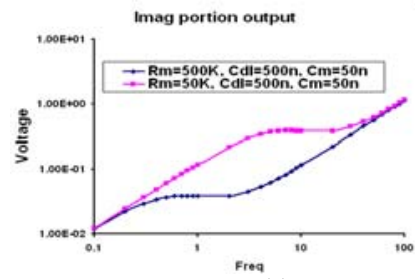

(a)

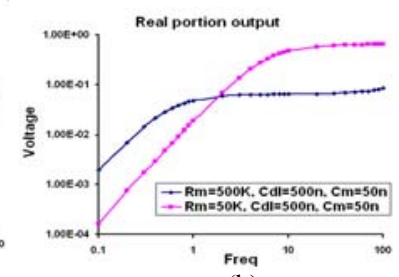

(b)
Figure 7. Simulated admittance measured by the new EIS system at different values of $\mathrm{R}_{\mathrm{M}}$, (a) imaginary portion and (b) real portion.

\section{REFERENCES}

[1] G. Krishna, J. Schulte, B. Cornell, R. Pace, and P. Osman, "Tethered Bilayer Membranes Containing Ionic Reservoirs: Selectivity and Conductance," Langmuir, vol. 19(6), pp. 2294-2305, 2003.

[2] R. Bragos, R. Blanco-Enrich, O. Casas, J. Rosell, "Characterisation of dynamic biologic systems using multisine based impedance spectroscopy", IEEE Conf. Instrumentation and Measurement Technology, Budapest, Hungary, vol. 1, pp. 44- 47, Jan 2001.

[3] S. Othman, E. Sacristan, C. Gonzalez, J. Pinzon, J. Aguado, P. Flores, et al,"In situ impedance spectroscopy of the intestinal mucosa in an ischemia-reperfusion model", IEEE Int. Conf. on Engineering in Medicine and Biology Society, Cancun, Mexico, vol. 4, pp. 32073210, Jan 2003.

[4] B. Hassler, R. Worden, A. Mason, P. Kim, N. Kohli, J. Zeikus, et al, "Biomimetic Interfaces for a Multifunctional Biosensor Array Microsystem,” IEEE Int. Conf. on Sensors, Vienna, Austria, pp. 991994, October 2004.

[5] G. Popkirov, R. Schindler, "A new impedance spectrometer for the investigation of electrochemical systems", Review of Scientific Instruments, vol. 63, pp. 5366- 5372, Nov 1992. 